\title{
Enhanced Piezoelectricity of Electrospun Polyvinylidene Fluoride Fibers for Energy Harvesting
}

\author{
Piotr K. Szewczyk, Arkadiusz Gradys, Sung Kyun Kim, Luana Persano, Mateusz Marzec, \\ Aleksandr Kryshtal, Tommaso Busolo, Alessandra Toncelli, Dario Pisignano, Andrzej Bernasik, \\ Sohini Kar-Narayan, Paweł Sajkiewicz, and Urszula Stachewicz*
}

Cite This: ACS Appl. Mater. Interfaces 2020, 12, 13575-13583

Read Online

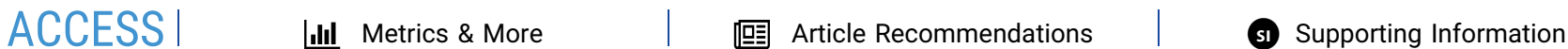

ABSTRACT: Piezoelectric polymers are promising energy materials for wearable and implantable applications for replacing bulky batteries in small and flexible electronics. Therefore, many research studies are focused on understanding the behavior of polymers at a molecular level and designing new polymer-based generators using polyvinylidene fluoride (PVDF). In this work, we investigated the influence of voltage polarity and ambient relative humidity in electrospinning of PVDF for energy-harvesting applications. A

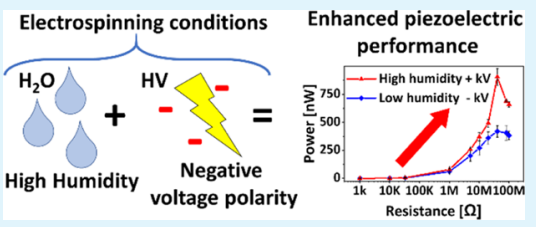
multitechnique approach combining microscopy and spectroscopy was used to study the content of the $\beta$-phase and piezoelectric properties of PVDF fibers. We shed new light on $\beta$-phase crystallization in electrospun PVDF and showed the enhanced piezoelectric response of the PVDF fiber-based generator produced with the negative voltage polarity at a relative humidity of $60 \%$. Above all, we proved that not only crystallinity but also surface chemistry is crucial for improving piezoelectric performance in PVDF fibers. Controlling relative humidity and voltage polarity increased the $d_{33}$ piezoelectric coefficient for PVDF fibers by more than three times and allowed us to generate a power density of $0.6 \mu \mathrm{W} \cdot \mathrm{cm}^{-2}$ from PVDF membranes. This study showed that the electrospinning technique can be used as a single-step process for obtaining a vast spectrum of PVDF fibers exhibiting different physicochemical properties with $\beta$-phase crystallinity reaching up to $74 \%$. The humidity and voltage polarity are critical factors in respect of chemistry of the material on piezoelectricity of PVDF fibers, which establishes a novel route to engineer materials for energy-harvesting and sensing applications.

KEYWORDS: PVDF, polymer crystallinity, electrospinning, piezoelectricity, voltage polarity

\section{INTRODUCTION}

The need for sustainable energy sources capable of meeting the ever-increasing energy demands of our world has spurred the development of energy-harvesting technologies such as piezoelectrics and triboelectrics. ${ }^{1}$ Current research in piezoelectric polymers is focused on wearable and flexible devices that could be incorporated into smart textiles such as biosensors, motion trackers, high-sensitivity acoustic sensors, and generators for always-on peripherals. Moreover, there is a growing need for piezoelectric polymers that could be used as active scaffolds for bioengineering applications. $^{2-4}$

Most of the mechanical energy is wasted; thus, several options have been studied to harness and convert it into other energy forms. Piezoelectric generators are materials capable of transforming mechanical stress into electricity. Many piezoelectric devices use ceramic materials such as barium titanate and lead zirconate titanate. These have exceptional energyharvesting efficiency; however, they are cost-intensive, brittle, and hard to process. ${ }^{5,6}$ Piezoelectric polymers are a promising alternative with polyvinylidene fluoride (PVDF) being the main candidate. PVDF combines high piezoelectric, pyroelectric, and ferroelectric properties with high mechanical strength, high thermal stability, and biocompatibility. ${ }^{7,8}$ With its unique properties, PVDF has found use in a broad spectrum of applications ranging from biomedical to energy-harvesting systems. It was successfully used as a membrane in protein detection systems, an electrolyte in rechargeable cells, part of rewritable memory, a substrate for crystalline ice growth, and cell scaffolds with controlled surface charge. ${ }^{9-12}$ PVDF can crystallize in at least four known phases which are related to different chain conformations and these are as follows: trans and gauche $\left(\mathrm{TG}^{+} \mathrm{TG}^{-}\right)$for $\alpha$ and $\delta$, all-trans (TTTT) for $\beta$, and $\left(\mathrm{T}_{3} \mathrm{G}^{+} \mathrm{T}_{3} \mathrm{G}^{-}\right)$for $\gamma^{7,13,14}$ The all-trans $\beta$-phase exhibits the highest piezoelectric response among all the PVDF phases because of its lack of symmetry in the distribution of negatively charged fluorine ions, which generates an electric dipole and thus a piezoelectric response. ${ }^{15}$ Numerous experimental techniques for obtaining high concentrations of the $\beta$-phase in PVDF have been investigated including mechanical stretching of polymer films, annealing, exposing to a strong

Received: February 10, 2020

Accepted: February 24, 2020

Published: February 24, 2020 



Figure 1. Schematic showing the principle of the electrospinning procedure used to investigate the humidity and voltage polarity effects on the surface chemistry of PVDF fibers.

electric field, ultrafast cooling rates during melt crystallization, and incorporation of additives. ${ }^{7,16-20}$ One of the most promising techniques is electrospinning combining electrical poling and mechanical stretching to produce a high content of the $\beta$-phase in PVDF. This fabrication process is often used in scaffold production for tissue engineering, ${ }^{21,22}$ energy harvesting, $^{23}$ filtration, ${ }^{24}$ water collection, ${ }^{25}$ production of hydrophobic materials, ${ }^{26,27}$ and many more. During the electrospinning process, the electric field applied to polymer solution introduces electrical polarization. The formation of the conejet introduces the mechanical stretching of the polymer jet to the counter electrode. Both electrical and mechanical effects present in electrospinning allow single-step acquisition of PVDF fibers rich in $\beta$-phase content. ${ }^{13,28,29}$ Among other parameters, the humidity during electrospinning was found to promote a higher fraction of the $\gamma$-phase in the solutiondeposited PVDF films, ${ }^{30}$ high film roughness, ${ }^{31}$ and changes in surface morphology of PVDF fibers, causing wrinkling. ${ }^{27,32}$ Similarly, wrinkling was observed in electrospun polymethyl methacrylate (PMMA) fibers and in polystyrene (PS), in which additionally, the internal porosity was present. ${ }^{33}$ In the case of alternating voltage polarity, the surface potential on PVDF fibers was controlled. ${ }^{8}$ Other research has confirmed that voltage polarity during electrospinning affected the composition of polycaprolactone and nylon 6 (PA6) electrospun fibers ${ }^{22,34}$ and was successfully used to enhance the triboelectric performance of PMMA fibers. ${ }^{35}$ Based on these findings, we propose that in PVDF, negative and positive voltage polarity allows us to interact with negatively charged fluorine groups present on the surface of the fibers and thus provides a tool to manipulate their surface chemistry and potential influencing the piezoelectric properties of fibers. As shown in Figure 1, the fluorine groups are repelled by negative voltage polarity and conversely can be attracted by the application of positive voltage polarity during electrospinning. Piezoelectricity of electrospun PVDF fibers was often correlated with a high degree of crystallinity, dimensional reduction of fibers, incorporation of additives, nonlinear extrinsic responses, poling, size, and chain orientation parallel to the surface; ${ }^{28,36,37}$ however, we propose that piezoelectricity can be modified with the two often missed parameters in electrospinning: voltage polarity and humidity. Thus, the aim of this study was to investigate the effect of voltage polarity and the ambient humidity during electrospinning on the PVDF chemistry by $\beta$-phase contents and piezoelectric response in PVDF fibers. We investigated the properties of both single fibers and the fiber network with a broad range of measurement techniques to perform a comprehensive study of PVDF properties across several length scales. Fiber morphology was investigated using scanning electron microscopy (SEM) and transmission electron microscopy (TEM). The chemical composition of the fibers surface was investigated using X-ray photoelectron spectroscopy (XPS). The phase composition of PVDF was measured using Fouriertransform infrared (FTIR) spectroscopy, differential scanning calorimetry (DSC), and wide-angle X-ray diffraction (WAXS). The piezoelectric response of single fibers and their topography were obtained from piezoresponse force microscopy (PFM), and the piezoelectric response of a fiber mesh was measured using a custom-built setup consisting of a linear motor and multimeter. Our work demonstrates the synergistic effect of nonstandard negative voltage polarity and high humidity conditions on piezoelectric performance of PVDF fibers showing a new, easily transferable approach for energyharvesting devices. The surface chemistry of PVDF can be controlled, leading to changes in the piezoelectric effect.

\section{EXPERIMENTAL SECTION}

Solution Preparation. A PVDF $\left(M_{\mathrm{w}}=275,000 \mathrm{~g} \mathrm{~mol}^{-1}\right.$, SigmaAldrich, UK) pellet was dissolved in a 1:1 solution of dimethylacetamide (DMAc, analytical standard, Avantor, Poland) and acetone (analytical standard, Avantor, Poland) to produce a polymer solution with a concentration of $24 \mathrm{wt} \%$. The solution was stirred for $4 \mathrm{~h}$ at a constant speed of $700 \mathrm{rpm}$ on a hot plate set to $50{ }^{\circ} \mathrm{C}$ (IKA RCT basic, Germany).

Electrospinning. Electrospinning of PVDF was carried out with the EC-DIG apparatus with the climate upgrade system (IME Technologies, The Netherlands). A constant voltage of $15 \mathrm{kV}$ with positive and negative polarity was applied to the stainless needle with an inner diameter of $0.8 \mathrm{~mm}$ located $18 \mathrm{~cm}$ from the grounded collector plate. The flow rate of the solution for all samples was set to $6 \mathrm{~mL} \cdot \mathrm{h}^{-1}$, and the time of electrospinning was $8 \mathrm{~min}$ in each case. Ambient temperature during electrospinning was kept constant at 25 ${ }^{\circ} \mathrm{C}$ and humidities of 30 and $60 \%$ were used. The produced fibers were collected on a copper grid with holey carbon films for TEM study and on silicon wafers coated with a $10 \mathrm{~nm}$ layer of gold for XPS and PFM. For SEM imaging and DSC and WAXS studies, fibers were electrospun directly on Al foil. Prior to SEM imaging, the electrospun fibers were coated with a $3 \mathrm{~nm}$ layer of gold using a rotary-pump sputter coater (Q150RS, Quorum Technologies, UK).

FTIR samples were prepared with electrospinning on paper frames attached directly to the steel drum. Finally, the piezoelectric generator mesh was electrospun directly on aluminum foil which served as one of the electrodes in the experiment.

Characterization Techniques. Using SEM (Merlin Gemini II, Zeiss, Germany), the samples were imaged with an accelerating voltage of $3 \mathrm{kV}$ and $150 \mathrm{pA}$ current at a working distance of $3-8 \mathrm{~mm}$. The measurements of the fiber diameter were performed utilizing Fiji (ImageJ, v1.51, NIH, USA) software, and 100 measurements of individual fibers for each type of sample were carried out by drawing a perpendicular line across the fiber to obtain the fiber diameter values. ${ }^{38}$ After freeze-fracturing fibers in liquid nitrogen, samples were fixed using carbon tape to stubs. TEM (Tecnai G2 20 Twin, ThermoFisher, USA) was used to investigate the morphology of PVDF fibers with bright field imaging at an accelerating voltage of 200 $\mathrm{kV}$. 
Humidity $30 \%$
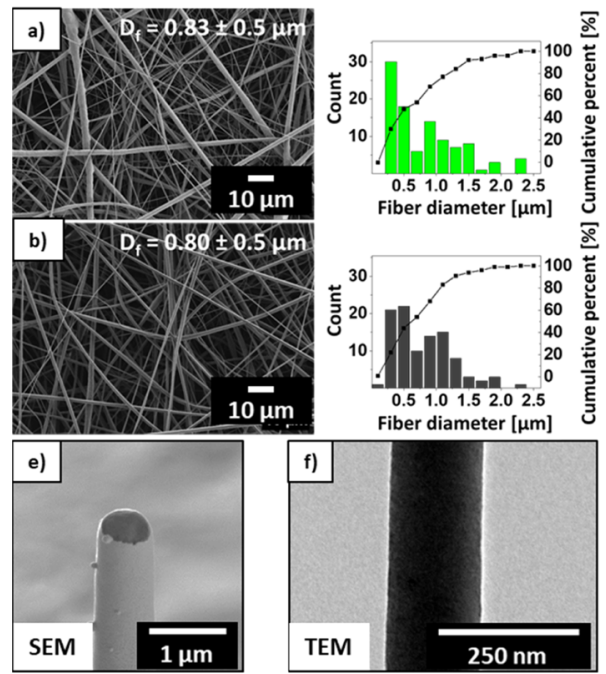

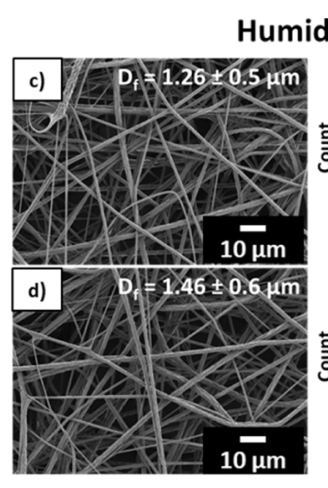

Humidity $60 \%$
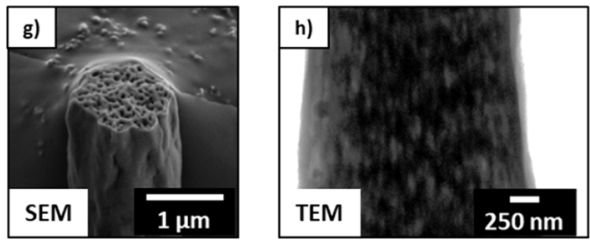

Figure 2. SEM micrographs and corresponding histograms including the curve showing the cumulative percentage of fiber diameters for all the PVDF samples: (a) PVDF30+, (b) PVDF30-, (c) PVDF60+, and (d) PVDF60-. The average diameter of fibers $\left(D_{\mathrm{f}}\right)$ with standard deviation values is indicated on SEM images. Representative images of all samples from freeze-fracture cross-sections of fibers (e) PVDF30+ and (g) PVDF60+ in comparison to TEM images (f) PVDF30+ and (h) PVDF60+.

The angle-resolved XPS analysis was carried out using a scanning VersaProbe II XPS system (PHI, USA) with monochromatic radiation from aluminum $\mathrm{K} \alpha(1486.6 \mathrm{eV})$ focused to a $100 \mu \mathrm{m}$ spot. The photoelectron take-off angle was set to $10^{\circ}$ for surface information, and the pass energy in the analyzer was set to $23.50 \mathrm{eV}$ to obtain high-energy resolution spectra for the $\mathrm{C} 1 \mathrm{~s}$ and $\mathrm{F} 1 \mathrm{~s}$ regions. The aligned fibers were measured perpendicular to the analyzer entrance; thus, in such a way, the curvature of the material was not affecting angle-resolved measurements. A dual-beam charge compensation with $7 \mathrm{eV} \mathrm{Ar}^{+}$ions and $1 \mathrm{eV}$ electrons was used to maintain a constant sample surface potential regardless of the sample conductivity. The operating pressure in the analytical chamber equals $4 \times 10^{-9}$ mbar. Deconvolution of spectra was carried out using MultiPak software (PHI, USA). The spectrum background was subtracted using the Shirley method.

WAXS investigations of crystal phases were performed using a D8 DISCOVER high-resolution X-ray diffractometer (Bruker, USA) equipped with a 1-D position-sensitive Lynx Eye detector. The monochromatic radiation of wavelength $\lambda=0.154 \mathrm{~nm}$ was used. The optics used were a parallel Goebel mirror, $1 \mathrm{~mm}$ circular slit, and 1 $\mathrm{mm}$ collimator. Fiber mats were removed from $\mathrm{Al}$ foil, folded to a thickness of $0.5 \mathrm{~mm}$ for high output and better statistics, and examined in the transmission mode at $21^{\circ} \mathrm{C}$. The time of registration was $1 \mathrm{~s}$ per point with a resolution of $2 \theta=0.02^{\circ}$. For comparison, the sample consisting of the native PVDF pellet was examined. In order to determine sample phase content, before deconvolution of the peaks, profiles were subtracted using a background scan and second-order polynomial. DSC measurements were carried out using a Pyris 1 differential scanning calorimeter (PerkinElmer, USA) equipped with Intracooler $2 \mathrm{P}$. Thermal analysis was performed at a heating rate of 10 $\mathrm{K} \cdot \mathrm{min}^{-1}$. For statistics, five separate samples, weighing $10 \mathrm{mg}$ each, were examined. IR spectroscopy was performed with an FTIR 6800 TypeA spectrophotometer (Jasco, USA) to investigate the overall crystallinity of PVDF fibers and to distinguish the precipitated phases.

The atomic force microscopy (AFM) based investigations were carried out using MultiMode 8 (Bruker, USA). For the investigation, all PVDF fibers were deposited on the gold-coated silicon substrate. The piezoresponse of the PVDF fibers was confirmed by lateral PFM. The internal lock-in amplifier was used to detect the piezoresponse signal equipped with conductive $\mathrm{Pt} / \mathrm{Cr}$-coated silicon tips (spring constant $3 \mathrm{~N} \mathrm{~m}^{-1}$ ), operating in the contact mode for imaging of topography and relative polarization by lateral PFM. For ferroelectricinduced dipole orientation measurement, the PFM signal was measured without the poling process in the AFM system. The piezoelectric $d_{33}$ coefficient in terms of electromechanical response was measured as a function of the applied AC bias which was applied between the AFM tip and the electrode deposited on the sample bottom surface. The change in PFM amplitude was measured for the applied AC bias from 2 to $10 \mathrm{~V}$. Calibration was carried out based on periodically poled lithium niobate $\left(\mathrm{LiNbO}_{3}\right.$, Bruker, USA) for which the piezoelectric coefficient is $7.5 \mathrm{pm} \cdot \mathrm{V}^{-1}$, for details, see Figure $6 \mathrm{Sa}$ in the Supporting Information. The slope of the resultant trend $\left(0.61554 \mathrm{mV} \cdot \mathrm{V}^{-1}\right)$ and piezoelectric coefficient of $\mathrm{LiNbO}_{3}$ allowed us to calculate the vertical sensitivity of our instrumentation as 12.185 $\mathrm{pm} \cdot \mathrm{mV}^{-1}$. PVDF fiber samples were measured in the same manner and adjusted to the calculated sensitivity, for details, see Figure $6 \mathrm{Sb}$ in the Supporting Information. ${ }^{39-41}$

Piezoelectric response from the bulk was investigated using the custom-built setup based on a linear motor. The sample was a $15 \times 10$ $\mathrm{mm}$ nanofiber network, placed between $\mathrm{Al}$ electrodes and connected with copper wires. One side of the generator was fixed to the nonconductive glass slide, while the other was isolated from the environment with insulating tape creating a closed system. The energy-harvesting measurements were conducted by placing the sample onto a fixed stage and cyclically tapping it with an arm attached to a linear motor. The arm was a $15 \times 10 \mathrm{~mm}$ punching plate matching precisely the size of the sample, ensuring uniform force distribution across the contact area. The cyclic forces tested were 1, 5, 10,15 , and $20 \mathrm{~N}$ with $1 \mathrm{~Hz}$ frequency to ensure reliable readings, and for power measurements, $20 \mathrm{~N}$ force at $1 \mathrm{~Hz}$ frequency was used. The voltage and current were measured across resistances from $1 \mathrm{k}$ to 100 M $\Omega$. Voltage measurements were performed by connecting a multimeter (Keithley 2002, Keithley Instruments, USA) in parallel, whereas current data were recorded using a picoammeter (Keithley 6487, Keithley Instruments, USA) in series. The schematics of the setup are presented in Figure 6.

\section{RESULTS AND DISCUSSION}

In this study, we evaluated the effect of voltage polarity $( \pm)$ and humidity $(30 / 60 \%)$ during electrospinning. This method allowed us to control the surface and bulk properties of fibers using the one-step approach, removing the need for any post electrospinning treatment. The PVDF fibers were manufactured via electrospinning, as shown in Figure 1. In the following, electrospun fibers produced with $60 \%$ humidity are 
a)

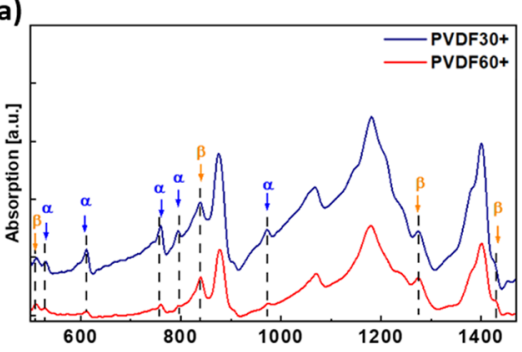

b)
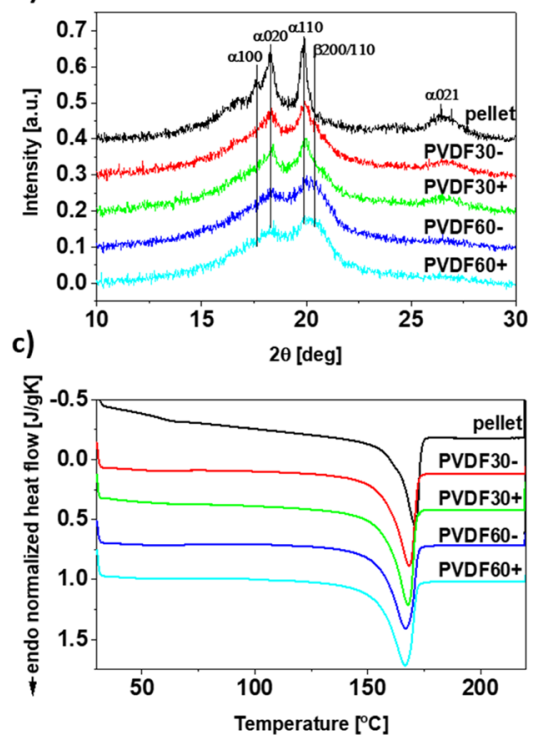

Figure 3. Characterization of electrospun PVDF fibers: (a) FTIR spectra of PVDF30+ and PVDF60+ samples, with data vertically shifted for better comparison, (b) WAXS profiles registered in transmission. The diffraction peaks belonging to the $\alpha$-phase, (100, $020,110$, and 021$)$ along with those of the $\beta$-phase $(200 / 110)$ are labeled, and (c) DSC heating scans.

labeled as PVDF60+ and PVDF60- for positive and negative polarity, respectively, whereas samples produced with $30 \%$ humidity are labeled as PVDF30+ and PVDF30-, respectively. The sign of the used polarity refers to the bias applied to the electrospinning needle with respect to the grounded collector. The fibers' morphology and diameter histograms are shown in Figure 2, confirming the previous results. ${ }^{26}$

We purposely produced only randomly oriented fibers as we want to minimize the additional mechanical stretching effect caused in aligned PVDF fibers by the rotating collector as it was reported to increase crystallinity. ${ }^{7,16,19,42,43}$ In the case of fibers obtained at $60 \%$ humidity, the wrinkled surface morphology was observed for both voltage polarities, and the average fiber diameter was similar: $1.26 \pm 0.5 \mu \mathrm{m}$ for PVDF60+ and $1.46 \pm 0.61 \mu \mathrm{m}$ for PVDF60- samples. On average, PVDF30 fibers yielded about $0.5 \mu \mathrm{m}$ smaller diameters than PVDF60 fibers with $0.83 \pm 0.53 \mu \mathrm{m}$ and $0.80 \pm 0.45 \mu \mathrm{m}$ for PVDF30+ and PVDF30-, respectively, and theses fibers had a smooth surface. Our findings are in line with results obtained for different sets of solvents such as dimethylacetamide and acetone and the dimethylacetamide/ tetrahydrofuran/acetone mixture where humidity affected the structure of the obtained PVDF fibers. Low humidity conditions yielded smooth uniform fibers and higher humidity led to wrinkling and porosity, indicating that our findings are applicable for different solvents too. ${ }^{29,32}$ We believe that in our


Figure 4. PFM scans of individual PVDF fibers showing (a) AFM topography and LPFM images, (b) and summary of the PFM amplitude for samples produced at $60 \%$ humidity $( \pm)$ and $30 \%$ humidity $( \pm)$.

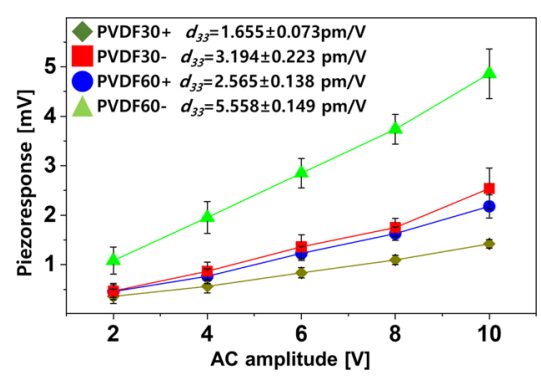

Figure 5. Piezoelectric $d_{33}$ coefficient plot of single PVDF fibers as measured with PFM. Measurements represented in this plot are shown in Figure 6S of the Supporting Information.

case, water from the humid environment was absorbed into the jet and acted as a nonsolvent for PVDF during electrospinning. Thus, vapor-induced phase separation preceded solidification because of a much slower evaporation rate of DMAc, which was the main solvent. Therefore, the internal voids and surface wrinkling for PVDF60 fibers without the presence of open porosity were observed similarly to previous studies on electrospun PS and PVDF fibers. ${ }^{29,44}$

Cumulative percent calculations (continuous curves in the histograms in Figure $2 a-d$ ) showed that for all the samples, fibers below $1.5 \mu \mathrm{m}$ constitute more than $50 \%$ of the entire population of the produced polymer fibers. No clear differences in the morphology of fibers were found when comparing samples prepared at different voltage polarities. The TEM investigation of fibers and SEM of a freeze-fractured cross-section of fibers revealed the internal porosity of PVDF60+ fibers in opposition to PVDF30+. These results showed the straightforward effect of humidity on fibers' internal structure and surface morphology. In fact, the 
a)

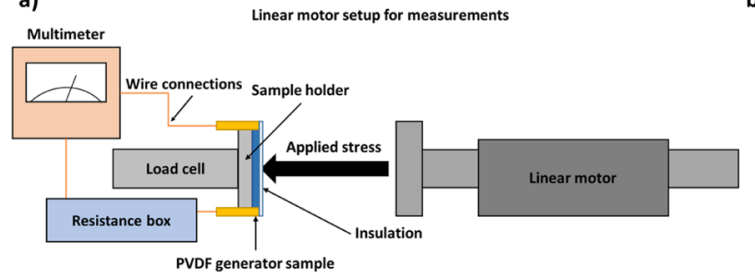

b)

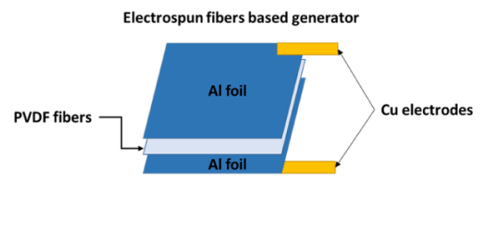

Figure 6. Schematic representation of the (a) piezoresponse measurement setup and (b) generator from randomly oriented PVDF mats.

Table 1. Summary of Crystallinity and Chemical Composition Measurements of all the PVDF Samples from WAXS, DSC, FTIR, and XPS ${ }^{a}$

\begin{tabular}{|c|c|c|c|c|c|c|c|c|c|c|c|}
\hline \multirow[b]{2}{*}{ sample } & \multicolumn{4}{|c|}{ WAXS } & \multicolumn{2}{|c|}{ DSC } & \multicolumn{3}{|c|}{ DSC calculated crystallinity } & \multirow{2}{*}{$\frac{\text { XPS }}{\text { F/C }}$} & \multirow{2}{*}{$\frac{\text { FTIR }}{F(\beta)[\%]}$} \\
\hline & $\overline{x_{\alpha}[\%]}$ & $x_{\beta}[\%]$ & $x_{c}[\%]$ & $x_{\beta} / x_{\alpha}$ & $T_{\mathrm{m}}\left[{ }^{\circ} \mathrm{C}\right]$ & $\Delta H_{\mathrm{m}}\left[\mathrm{J} \cdot \mathrm{g}^{-1}\right]$ & $x_{\alpha}[\%]$ & $x_{\beta}[\%]$ & $x_{\mathrm{c}}[\%]$ & & \\
\hline PVDF pellet & 34.6 & 0 & 34.6 & 0 & $171 \pm 0.2$ & $38.6 \pm 0.2$ & $36.9 \pm 0.2$ & 0 & $36.9 \pm 0.2$ & & \\
\hline PVDF30- & 21.7 & 7 & 28.7 & 0.32 & $168.5 \pm 0.5$ & $53.1 \pm 0.6$ & $29.9 \pm 0.3$ & $10.0 \pm 0.1$ & $39.9 \pm 0.4$ & 0.83 & 50 \\
\hline PVDF30+ & 21.5 & 7.3 & 28.8 & 0.34 & $167.7 \pm 0.3$ & $54.7 \pm 0.9$ & $30.3 \pm 0.5$ & $10.4 \pm 0.2$ & $40.7 \pm 0.7$ & 0.88 & 52 \\
\hline PVDF60- & 12.2 & 14.2 & 26.4 & 1.16 & $166.8 \pm 0.2$ & $56.8 \pm 0.2$ & $15.8 \pm 0.1$ & $18.3 \pm 0.1$ & $34.1 \pm 0.2$ & 0.78 & 70 \\
\hline PVDF60+ & 12.9 & 14.1 & 27 & 1.09 & $166.6 \pm 0.3$ & $56.2 \pm 0.4$ & $16.3 \pm 0.1$ & $17.8 \pm 0.1$ & $34.1 \pm 0.2$ & 0.89 & 74 \\
\hline
\end{tabular}

${ }^{a}$ Details on crystallinity calculation from WAXS and DSC are provided in the Supporting Information. In the case of the WAXS results, the absolute maximum error is $3 \%$ and between the samples may be treated as negligible (see deconvolution details in the Supporting Information).

environmental conditions during electrospinning affect the evaporation rate of solvents from the solution and determine the final morphology and properties of the obtained polymer fibers. ${ }^{45,46}$ Differences in the morphology of PVDF60 and PVDF30 fibers were related to water penetration during the time of the liquid jet, which caused polymer expansion, leading to the higher average diameter and porous structure of fibers obtained at $60 \%$ humidity, indicated in Figure 2a,b,g,h. A similar effect has been previously reported for hydrophobic polymers. $^{44,45,47}$ Importantly, PVDF fibers electrospun from DMF/acetone solution followed the same trend, indicating a similar effect regardless of the used solvents. ${ }^{48}$

The phase composition of PVDF was investigated using FTIR. The FTIR spectra of fibers electrospun with positive voltage polarity at different humidity conditions are shown in Figure 3a. Spectra comparing fibers electrospun at different voltage polarities can be found in Figure $1 S$ in the Supporting Information. The polar $\beta$-phase absorption bands at 510, 840, 1274 , and $1430 \mathrm{~cm}^{-1}$ appear distinctly in all the samples, while the typical features of the nonferroelectric $\alpha$-phase (530, 612, 760,796 , and $970 \mathrm{~cm}^{-1}$ ) are clearly indicated in the samples obtained at lower ambient humidity (PVDF30 samples). The relative fraction of the $\beta$-phase for each sample was estimated by analyzing FTIR results according to the following equation $^{49}$

$$
F(\beta)=A_{\beta} /\left(1.26 A_{\alpha}+A_{\beta}\right)
$$

where $A_{\alpha}$ and $A_{\beta}$ indicate the intensities of the FTIR peaks at $760 \mathrm{~cm}^{-1}$ and $840 \mathrm{~cm}^{-1}$, respectively. This approach was chosen based on previous research on PVDF crystallinity. A factor of 1.26 for the $\alpha$-phase is added to compensate the difference in the absorption coefficients, which are $6.1 \times 10^{4}$ and $7.7 \times 10^{4} \mathrm{~cm}^{2} \cdot \mathrm{mol}^{-1}$ for $\alpha$ and $\beta$ phases, respectively. ${ }^{50,51}$

PVDF60 samples exhibit about 20\% higher amounts of the $\beta$-phase when compared to their PVDF30 counterparts for both positive and negative voltage polarities, giving 74 and $70 \%$ for PVDF60+ and PVDF60- compared to 52 and 50\% for PVDF30+ and PVDF30-, respectively. Zaarour et al. reached $87.5 \%$ of the $\beta$-phase for PVDF electrospun from
DMF/THF/acetone solution. ${ }^{29}$ Higher crystallinity values in the samples processed at $60 \%$ humidity are associated to water priming on the surface of the polymer jet, which causes a longitudinal deformation of polymer chains, observed in wrinkling of fibers (Figure $2 \mathrm{~g}, \mathrm{~h}$ ). This effect increases the content of $\beta$ crystallites. ${ }^{37}$ The $\beta$-phase orientation is influenced also by stress, ${ }^{19}$ which happens during pore formation inside PVDF60 fibers. Additionally, we investigated the influence of the voltage polarity in electrospinning on the $\beta$-phase. Results highlight a modest increase of the $\beta$-phase content for fibers electrospun with positive polarity, namely, by 2 and $3 \%$ for fibers electrospun at 30 and $60 \%$ of humidity, respectively.

WAXS and DSC techniques were used to investigate further the phase composition and thermal characteristics of electrospun PVDF fibers. In the WAXS profile for a PVDF pellet, shown in Figure $3 \mathrm{~b}$, only the $\alpha$-phase is present; therefore, it is used as a reference for other phases present in electrospun PVDF. The WAXS profiles registered for electrospun fibers show clearly a much lower intensity at positions of the $\alpha$-phase diffraction peaks. WAXS profiles for PVDF60 samples evidence more of the $\beta$-phase than profiles for PVDF30 samples, indicated by a lower intensity of the $\alpha 021$ diffraction peak at $2 \theta=26^{\circ}$ and a higher intensity of the $\beta 200 / 110$ diffraction peak at $2 \theta=20.4^{\circ}$. In order to reveal quantitatively the effect of humidity and polarity on the $\beta$-phase content, deconvolution of the WAXS profiles was performed. Values of the $\alpha$ - and $\beta$-phase contents are collated in Table 1 along with all the corresponding deconvoluted profiles. The deconvolution procedure details are provided in the Supporting Information in Figure 3S.

The DSC heating scans presented in Figure $3 \mathrm{c}$ show only a single endothermic peak related to melting of the crystal phases for each sample. The higher melting temperature was observed for high content of $\alpha$-phase samples: a pellet and PVDF30 fibers. Moreover, for a PVDF pellet and fibers spun at low humidity, PVDF30, which are of high content of the $\alpha$ phase, the melting peak is much narrower than for fibers with higher $\beta$-phase content, that is, electrospun at high humidity, PVDF60. The temperature of the melting peak, $T_{\mathrm{m}}$, and the 
melting heat, $\Delta H_{\mathrm{m}}$, determined from the DSC scans are shown in Table 1. From these data, it is seen that the higher the $\beta$ phase content, the higher the melting heat, $\Delta H_{\mathrm{m}}$, and lower the melting temperature, $T_{\mathrm{m}}$. These results seem very reasonable as the $\beta$-phase is considered as the low-temperature phase of PVDF; however, its equilibrium melting temperature, $T_{\mathrm{m}}{ }^{0}$, is not known. Moreover, it is generally accepted that the $\beta$-phase, because of strong polar interactions between parallel dipoles existing in the TTT conformation, should have much higher melting enthalpy than the $\alpha$-phase. In the literature, the melting enthalpy of the $\beta$-phase is reported as ca. $220 \mathrm{~J} \cdot \mathrm{g}^{-1}$ in comparison to $104.5 \mathrm{~J} \cdot \mathrm{g}^{-1}$ for the $\alpha$-phase, ${ }^{52}$ which was very often used in previous studies showing high $\beta$-phase content in PVDF.

Our results confirm higher melting enthalpy of the $\beta$-phase as obtained values of the melting heat increase with the increase of the $\beta$-phase content. With the melting enthalpies of the $\alpha$ and $\beta$ phases assumed as 104.5 and $220 \mathrm{~J} \cdot \mathrm{g}^{-1}$,, 2 respectively, and taking the $x_{\beta} / x_{\alpha}$ ratio as determined from WAXS, we calculated $x_{\alpha}$ and $x_{\beta}$ from the melting heat, $\Delta H_{\mathrm{m}}$, as measured by DSC. Calculated $x_{\alpha}, x_{\beta}$, and $x_{\mathrm{c}}$ values for fibers and a pellet were found linearly related to WAXS results, see Table 1. This means that the phase content values from WAXS and the characteristic melting heat values from DSC are well correlated. However, higher content values were obtained from the melting heat (DSC) than from WAXS. This outcome is due to the higher sensitivity of the DSC method. The thermal effects in DSC are arising from melting of imperfect crystals, which are excluded in the WAXS method. Additionally, explanation for the higher phase content, as determined from DSC, is related to hidden recrystallization and crystal improvement occurring during the DSC heating scan, especially when highly imperfect crystals are present in fibers, resulting in a substantial increase in the size of the final melting peak. The plot of calculated crystallinity from DSC versus crystallinity from WAXS is included in the Supporting Information, Figure $4 S$.

The results from WAX, DSC, XPS, and FTIR presented in Table 1 highlight an increase of the $\beta$-phase for PVDF fibers electrospun with positive voltage polarity compared to those electrospun with the negative voltage polarity. These changes were induced by either repelling negatively charged fluorine groups at the surface of PVDF fibers, when using negative charges, or attracting them when using positive voltage polarity. This was confirmed with XPS results summarized in Table 1. A similar effect of voltage polarity influence on the reorientation of the functional groups at the surface of electrospun fibers was observed for oxygen in the case of PA6. $^{34}$

The controlled humidity during electrospinning has the strongest effect on the phase content in the electrospun PVDF. The highest $\beta$-phase content of $14 \%$ was observed for PVDF60, and for PVDF30, it was two times lower (at 7\%). Additionally, slightly higher overall crystallinity, $x_{\mathcal{c}}$, was obtained at a humidity of $30 \%$. Voltage polarity had a minor effect on the phase content, as seen in the WAXS, DSC, and FTIR results, Figure 3. However, the voltage polarity caused a significant change in the surface chemistry of PVDF fibers, as indicated by XPS, performed at the grazing angle, thus giving chemical content from an approximate depth of $2 \mathrm{~nm}$. The investigated fluorine to carbon ratio (F/C) showed that the highest amount of $\mathrm{F}^{-}$at. $\%$ at the fiber surface was obtained for PVDF60+ in opposition to the lowest amount measured for
PVDF60-, confirming the previous results. ${ }^{8}$ In all cases, PVDF fibers showed higher $\mathrm{F}^{-}$content at the fibers' surface when electrospun with positive voltage polarity because the conditions lead to attraction of negatively charged $\mathrm{F}^{-}$groups toward the fiber surface. The fluorine to carbon ratio, F/C ratio, in PVDF is presented in Table 1, and all the XPS spectra are presented in Figure 5S, including detailed values in Table $1 S$ and exemplary WESS spectra of PVDF60+ in Figure $2 S$ in the Supporting Information. Voltage polarity affected the surface chemistry regardless of the relative humidity during electrospinning of PVDF.

To verify the effect of surface chemistry and the bulk of PVDF fibers on their properties, their piezoelectric performance was investigated. First, we carried out measurements highlighting the piezoelectric effect using PFM. In Figure 4, the topography, PFM amplitude, and phase images of PVDF fibers are displayed. PFM was performed on fibers with a comparable diameter $(\sim 2 \mu \mathrm{m})$ under equal environmental conditions to enable comparison of the PFM amplitudes affected by the voltage polarity during electrospinning. The graphical representation of PFM results in Figure $4 \mathrm{~b}$ indicates that negative voltage polarity during electrospinning leads to enhanced piezoelectric response in both cases. Despite higher overall crystallinity, the PVDF60+ fibers exhibit lower PMF amplitude than PVDF30-, which was caused by the voltage polarity affecting fibers' surface properties. The PVDF60fibers yielded the best results by combining high $\beta$-phase content, higher overall crystallinity, and modified surface chemistry. Importantly, the PFM phases of PVDF60+ and PVDF30+ have had a similar polarization direction. In the PFM measurement, we focused on the top part of the PVDF fibers for the comparison of the same contact area between the AFM probe and the sample surface. Moreover, the polarization of PVDF fibers obtained at $60 \%$ humidity showed a more dominant direction than that of fibers obtained at $30 \%$ humidity as the PVDF60+ and PVDF60- exhibit a higher poling effect as a relatively high concentration of the $\beta$-phase was measured. Therefore, both surface chemistry and structural properties such as crystallinity were affecting the piezoelectric performance of PVDF fibers.

Confirming PFM amplitude results in Figure 4, the $d_{33}$ piezoelectric coefficient measurements show the increase from 1.655 to $2.565 \mathrm{pm} \cdot \mathrm{V}^{-1}$ for PVDF30+ and PVDF60+, respectively. More importantly, with negative voltage polarity, we observe a sharp increase to 3.194 and $5.558 \mathrm{pm} \cdot \mathrm{V}^{-1}$ for PVDF30- and PVDF60-, respectively. As observed with other techniques, PVDF60- fibers show exceptional performance when compared to other PVDF samples. Additionally, PVDF60- fibers performed more than two times better than its PVDF60+ counterpart, showing the synergistic effect of humidity and voltage polarity on piezoelectric properties of electrospun fibers.

Furthermore, the piezoresponse from the fiber network was investigated using a custom-built setup shown in Figure 6. In Figure $7 \mathrm{a}$, the power measurement results are presented for all PVDF generators prepared from PVDF fibers in the form of mats. The highest power output was recorded for the sample PVDF60-. This result confirmed PFM results as the PVDF60- sample was found to have the highest piezoelectric response from the surface, see Figures 4 and 5. The PVDF60generator had a maximum average power output of $0.91 \mu \mathrm{W}$ at a load resistance of $40 \mathrm{M} \Omega$, whereas PVDF60+ had a maximum average output of $0.58 \mu \mathrm{W}$ at $100 \mathrm{M} \Omega$, under the 
a)

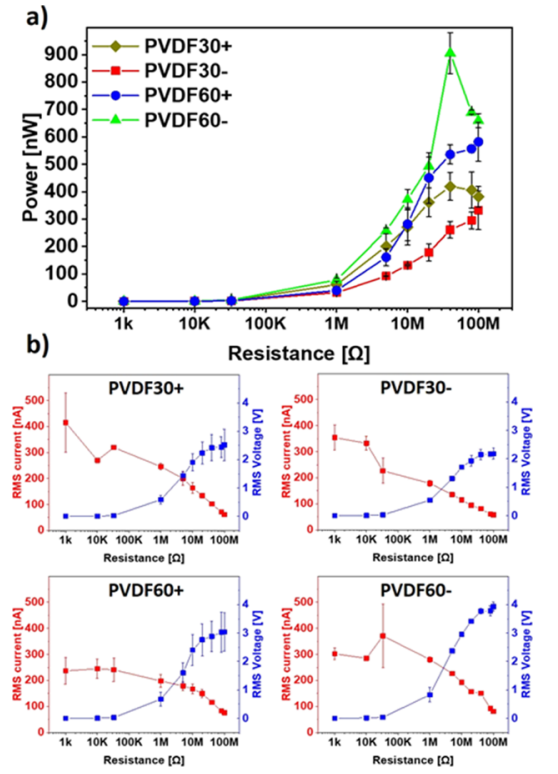

Figure 7. Piezoelectric performance of generators built from electrospun PVDF fibers produced at $H=30 \%$ (PVDF30) and at $H=60 \%$ (PVDF60): (a) power output of different PVDF generators. (b) rms voltage and current of all tested PVDF samples across load resistances from $1 \mathrm{k}$ to $100 \mathrm{M} \Omega$.

testing conditions used. For PVDF30 generators, the maximum outputs were $0.42 \mathrm{~W}$ and $0.33 \mu \mathrm{W}$ at 40 and $100 \mathrm{M} \Omega$ resistances for PVDF30+ and PVDF30-, respectively. The PVDF60- membrane, therefore, produced a maximum output power that was three times higher than that of the PVDF30device and more than $0.32 \mu \mathrm{W}$ higher than PVDF60+. Fibers prepared at $60 \%$ humidity were found to generate significantly higher amounts of power. At $100 \Omega$, the PVDF60+ sample produced 200.4 nW more power than PVDF30+ and similarly, PVDF60- produced 326.5 more power than PVDF30-. The power density outputs of the mats were $0.60,0.39,0.28$, and $0.22 \mu \mathrm{W} \cdot \mathrm{cm}^{-2}$ for PVDF60-, PVDF60+, PVDF30+, and PVDF30-, respectively. Our mats show an exceptional output compared to the literature and our devices at right conditions generated up to about $660 \mathrm{nW}$, whereas the measured generated power values are generally in the range of 10-100 nW. ${ }^{53,54}$ It should be noted that the direct power output comparison of PVDF mats is challenging because of the variation in mat thickness and porosity between studies. Regarding the fatigue tests, the PVDF fibers showed even better mechanical stability than already tested fiber-based power generators. ${ }^{35}$ Piezoresponse measurements confirmed results from PFM and shed new light on the usage of voltage polarity and humidity as key factors to tailor chemistry and the piezoelectric performance of electrospun PVDF fibers.

\section{CONCLUSIONS}

We proposed a single-step method to enhance surface chemistry and the piezoelectric performance of electrospun PVDF fibers that can be controlled via voltage polarity and relative humidity during electrospinning. The piezoelectric response of PVDF fibers was measured and correlated to surface chemical composition analyzed with XPS. We also included extensive SEM and TEM studies of surface and internal structure of fibers giving comprehensive insight into the PVDF fibers obtained with different processing conditions.
Importantly, we observed that humidity affects the internal structure of fibers and its phase composition regardless of the surface chemistry that can be controlled via voltage polarity. The spectroscopy investigation proved that higher humidity during electrospinning leads to higher $\beta$-phase content in fibers. These results were confirmed by WAXS and DSC, which showed almost two times higher concentrations of the $\beta$-phase in PVDF60 samples compared to PVDF30. Therefore, we confirmed that electrospinning at higher humidity leads to higher amounts of the $\beta$-phase in electrospun fibers. Importantly, higher humidity was correlated with a higher PFM amplitude and $d_{33}$ coefficient; thus, PVDF60- fibers were the most piezoelectric of all samples because of the combination of higher humidity and negative voltage polarity effects exhibiting the $d_{33}$ coefficient as high as $5.558 \mathrm{pm} \cdot \mathrm{V}^{-1}$ and generated up to $0.60 \mu \mathrm{W} \cdot \mathrm{cm}^{-2}$ of power when used as a generator. This unique study showed the importance of manufacturing settings and their impact on desired properties of fibers in electrospinning which can be applied in energyharvesting devices and beyond.

\section{ASSOCIATED CONTENT}

Supporting Information

The Supporting Information is available free of charge at https://pubs.acs.org/doi/10.1021/acsami.0c02578.

FTIR spectra; wide energy survey scan for PVDF60+ fibers; deconvoluted WAXS profiles; crystallinity data; XPS spectra; PFM data; and grazing angle XPS results (PDF)

\section{AUTHOR INFORMATION}

\section{Corresponding Author}

Urszula Stachewicz - International Centre of Electron Microscopy for Materials Science and Faculty of Metals Engineering and Industrial Computer Science, AGH University of Science and Technology, 30-059 Krakow, Poland; ○ orcid.org/0000-0001-5102-8685; Email: ustachew@ agh.edu.pl

\section{Authors}

Piotr K. Szewczyk - International Centre of Electron Microscopy for Materials Science and Faculty of Metals Engineering and Industrial Computer Science, AGH University of Science and Technology, 30-059 Krakow, Poland; (1) orcid.org/0000-0003-1441-7387

Arkadiusz Gradys - Polish Academy of Sciences, Institute of Fundamental Technological Research, 02-106 Warszawa, Poland

Sung Kyun Kim - Department of Materials Science and Metallurgy, University of Cambridge, CB3 OFS Cambridge, U.K.

Luana Persano - Nanoscience Institute NANO, Italian National Research Council (CNR), 56127 Pisa, Italy

Mateusz Marzec - Academic Centre for Materials and Nanotechnology, AGH University of Science and Technology, 30-059 Krakow, Poland; (i) orcid.org/0000-0001-9834-3930

Aleksandr Kryshtal - International Centre of Electron Microscopy for Materials Science and Faculty of Metals Engineering and Industrial Computer Science, AGH University of Science and Technology, 30-059 Krakow, Poland; (1) orcid.org/0000-0002-6528-8821 
Tommaso Busolo - Department of Materials Science and Metallurgy, University of Cambridge, CB3 OFS Cambridge, U.K.

Alessandra Toncelli - Nanoscience Institute NANO, Italian National Research Council (CNR), 56127 Pisa, Italy; Department of Physics, University of Pisa, 56127 Pisa, Italy

Dario Pisignano - Nanoscience Institute NANO, Italian National Research Council (CNR), 56127 Pisa, Italy; Department of Physics, University of Pisa, 56127 Pisa, Italy; - orcid.org/0000-0003-3758-5199

Andrzej Bernasik - Academic Centre for Materials and Nanotechnology and Faculty of Physics and Applied Computer Science, AGH University of Science and Technology, 30-059 Krakow, Poland

Sohini Kar-Narayan - Department of Materials Science and Metallurgy, University of Cambridge, CB3 OFS Cambridge, U.K.; 10 orcid.org/0000-0002-8151-1616

Pawel Sajkiewicz - Polish Academy of Sciences, Institute of Fundamental Technological Research, 02-106 Warszawa, Poland

Complete contact information is available at: https://pubs.acs.org/10.1021/acsami.0c02578

\section{Notes}

The authors declare no competing financial interest.

\section{ACKNOWLEDGMENTS}

U.S. and P.K.S. acknowledge the funding from the Sonata Bis 5 project granted by National Science Centre, no. 2015/18/E/ ST5/00230, and STSM Grant from COST Action CA17107, CONTEXT, funded by the European Commission, allowing the PFM measurements. A.G. and P.S. acknowledge the use of $\mathrm{CePT}$ infrastructure financed by the European Union-the European Regional Development Fund within the Operational Programme "Innovative economy" for 2007-2013 for WAX and DSC studies. S.K.-N. acknowledges financial support from the European Research Council through an ERC Starting Grant (grant no. ERC-2014-STG-639526, NANOGEN). T.B. acknowledges funding from the EPSRC Cambridge NanoDTC, EP/G037221/1. D.P. and A.T. acknowledge the support from the project PRA 201834 ("ANISE") from the University of Pisa and $\mathrm{J}$. $\mathrm{Xu}$ for the support during FTIR measurements.

\section{REFERENCES}

(1) Choi, Y. S.; Jing, Q.; Datta, A.; Boughey, C.; Kar-Narayan, S. A Triboelectric Generator Based on Self-Poled Nylon-11 Nanowires Fabricated by Gas-Flow Assisted Template Wetting. Energy Environ. Sci. 2017, 10, 2180-2189.

(2) Persano, L.; Dagdeviren, C.; Su, Y.; Zhang, Y.; Girardo, S.; Pisignano, D.; Huang, Y.; Rogers, J. A. High Performance Piezoelectric Devices Based on Aligned Arrays of Nanofibers of Poly(Vinylidenefluoride-Co-Trifluoroethylene). Nat. Commun. 2013, 4, 1633.

(3) Li, M.-y.; Bidez, P.; Guterman-Tretter, E.; Guo, Y.; MacDiarmid, A. G.; Lelkes, P. I.; Yuan, X.-b.; Yuan, X.-y.; Sheng, J.; Li, H.; et al. Electroactive and Nanostructured Polymers as Scaffold Materials for Neuronal and Cardiac Tissue Engineering. Chin. J. Polym. Sci. 2007, 25,331 .

(4) Lang, C.; Fang, J.; Shao, H.; Ding, X.; Lin, T. High-Sensitivity Acoustic Sensors from Nanofibre Webs. Nat. Commun. 2016, 7, 11108 .
(5) Parangusan, H.; Ponnamma, D.; Al-Maadeed, M. A. A. Stretchable Electrospun PVDF-HFP/Co-ZnO Nanofibers as Piezoelectric Nanogenerators. Sci. Rep. 2018, 8, 754.

(6) Chang, J.; Dommer, M.; Chang, C.; Lin, L. Piezoelectric Nanofibers for Energy Scavenging Applications. Nano Energy 2012, 1, 356-371.

(7) Ico, G.; Showalter, A.; Bosze, W.; Gott, S. C.; Kim, B. S.; Rao, M. P.; Myung, N. V.; Nam, J. Size-Dependent Piezoelectric and Mechanical Properties of Electrospun P(VDF-TrFE) Nanofibers for Enhanced Energy Harvesting. J. Mater. Chem. A 2016, 4, 2293-2304.

(8) Szewczyk, P. K.; Metwally, S.; Karbowniczek, J. E.; Marzec, M. M.; Stodolak-Zych, E.; Gruszczyński, A.; Bernasik, A.; Stachewicz, U. Surface-Potential-Controlled Cell Proliferation and Collagen Mineralization on Electrospun Polyvinylidene Fluoride (PVDF) Fiber Scaffolds for Bone Regeneration. ACS Biomater. Sci. Eng. 2019, 5, $582-593$.

(9) Braga, M. H.; M Subramaniyam, C.; Murchison, A. J.; Goodenough, J. B. Nontraditional, Safe, High Voltage Rechargeable Cells of Long Cycle Life. J. Am. Chem. Soc. 2018, 140, 6343-6352.

(10) Yonekuta, Y.; Susuki, K.; Oyaizu, K.; Honda, K.; Nishide, H. Battery-Inspired, Nonvolatile, and Rewritable Memory Architecture: A Radical Polymer-Based Organic Device. J. Am. Chem. Soc. 2007, 129, 14128-14129.

(11) Tran, T. N. N.; Cui, J.; Hartman, M. R.; Peng, S.; Funabashi, H.; Duan, F.; Yang, D.; March, J. C.; Lis, J. T.; Cui, H.; et al. A Universal DNA-Based Protein Detection System. J. Am. Chem. Soc. 2013, 135, 14008-14011.

(12) Rosa, L. G.; Xiao, J.; Losovyj, Y. B.; Gao, Y.; Yakovkin, I. N.; Zeng, X. C.; Dowben, P. A. Crystalline Ice Grown on the Surface of the Ferroelectric Polymer Poly(Vinylidene Fluoride) (70\%) and Trifluoroethylene (30\%). J. Am. Chem. Soc. 2005, 127, 17261-17265.

(13) Lei, T.; Zhu, P.; Cai, X.; Yang, L.; Yang, F. Electrospinning of PVDF Nanofibrous Membranes with Controllable Crystalline Phases. Appl. Phys. A 2015, 120, 5-10.

(14) Li, M.; Wondergem, H. J.; Spijkman, M.-J.; Asadi, K.; Katsouras, I.; Blom, P. W. M.; De Leeuw, D. M. Revisiting the $\delta$ Phase of Poly(Vinylidene Fluoride) for Solution-Processed Ferroelectric Thin Films. Nat. Mater. 2013, 12, 433-438.

(15) Wan, C.; Bowen, C. R. Multiscale-Structuring of Polyvinylidene Fluoride for Energy Harvesting: The Impact of Molecular-, Microand Macro-Structure. J. Mater. Chem. A 2017, 5, 3091-3128.

(16) Grubb, D. T.; Rich Kearney, F. Zone Drawing and Mechanical Relaxation of Poly(Vinylidene Fluoride). J. Polym. Sci., Part B: Polym. Phys. 1990, 28, 2071-2078.

(17) Gregorio, R., Jr.; Ueno, E. M. Effect of Crystalline Phase, Orientation and Temperature on the Dielectric Properties of Poly (Vinylidene Fluoride) (PVDF). J. Mater. Sci. 1999, 34, 4489-4500.

(18) Gradys, A.; Sajkiewicz, P.; Zhuravlev, E.; Schick, C. Kinetics of Isothermal and Non-Isothermal Crystallization of Poly(Vinylidene Fluoride) by Fast Scanning Calorimetry. Polymer 2016, 82, 40-48.

(19) Sajkiewicz, P.; Wasiak, A.; Gocłowski, Z. Phase Transitions during Stretching of Poly(Vinylidene Fluoride). Eur. Polym. J. 1999, 35, 423-429.

(20) Wang, J.; Li, H.; Liu, J.; Duan, Y.; Jiang, S.; Yan, S. On the $\alpha \rightarrow$ $\beta$ Transition of Carbon-Coated Highly Oriented PVDF Ultrathin Film Induced by Melt Recrystallization. J. Am. Chem. Soc. 2003, 125, 1496-1497.

(21) Agarwal, S.; Wendorff, J. H.; Greiner, A. Use of Electrospinning Technique for Biomedical Applications. Polymer 2008, 49, 56035621.

(22) Metwally, S.; Karbowniczek, J. E.; Szewczyk, P. K.; Marzec, M. M.; Gruszczyński, A.; Bernasik, A.; Stachewicz, U. Single-Step Approach to Tailor Surface Chemistry and Potential on Electrospun PCL Fibers for Tissue Engineering Application. Adv. Mater. Interfaces 2019, 6, 1801211.

(23) Jing, Q.; Kar-Narayan, S. Nanostructured Polymer-Based Piezoelectric and Triboelectric Materials and Devices for Energy Harvesting Applications. J. Phys. D: Appl. Phys. 2018, 51, 303001. 
(24) Rajesh, K. P.; Natarajan, T. S. Electrospun Polymer Nanofibrous Membrane for Filtration. J. Nanosci. Nanotechnol. 2009, 9, 5402-5405.

(25) Ganesh, V. A.; Ranganath, A. S.; Baji, A.; Raut, H. K.; Sahay, R.; Ramakrishna, S. Hierarchical Structured Electrospun Nanofibers for Improved Fog Harvesting Applications. Macromol. Mater. Eng. 2017, 302, 1600387.

(26) Szewczyk, P.; Ura, D.; Metwally, S.; Knapczyk-Korczak, J.; Gajek, M.; Marzec, M.; Bernasik, A.; Stachewicz, U. Roughness and Fiber Fraction Dominated Wetting of Electrospun Fiber-Based Porous Meshes. Polymers 2018, 11, 34.

(27) Szewczyk, P. K.; Knapczyk-Korczak, J.; Ura, D. P.; Metwally, S.; Gruszczyński, A.; Stachewicz, U. Biomimicking Wetting Properties of Spider Web from Linothele Megatheloides with Electrospun Fibers. Mater. Lett. 2018, 233, 211-214.

(28) Andrew, J. S.; Clarke, D. R. Enhanced Ferroelectric Phase Content of Polyvinylidene Difluoride Fibers with the Addition of Magnetic Nanoparticles. Langmuir 2008, 24, 8435-8438.

(29) Zaarour, B.; Zhu, L.; Huang, C.; Jin, X. Enhanced Piezoelectric Properties of Randomly Oriented and Aligned Electrospun PVDF Fibers by Regulating the Surface Morphology. J. Appl. Polym. Sci. 2019, 136, 47049.

(30) Benz, M.; Euler, W. B. Determination of the Crystalline Phases of Poly(Vinylidene Fluoride) under Different Preparation Conditions Using Differential Scanning Calorimetry and Infrared Spectroscopy. J. Appl. Polym. Sci. 2003, 89, 1093-1100.

(31) Szewczyk, P. K.; Metwally, S.; Krysiak, Z. J.; Kaniuk, Ł.; Karbowniczek, J. E.; Stachewicz, U. Enhanced Osteoblasts Adhesion and Collagen Formation on Biomimetic Polyvinylidene Fluoride (PVDF) Films for Bone Regeneration. Biomed. Mater. 2019, 14, 065006.

(32) Zaarour, B.; Zhu, L.; Huang, C.; Jin, X. Controlling the Secondary Surface Morphology of Electrospun PVDF Nanofibers by Regulating the Solvent and Relative Humidity. Nanoscale Res. Lett. 2018, 13, 285.

(33) Haider, A.; Haider, S.; Kang, I.-K. A Comprehensive Review Summarizing the Effect of Electrospinning Parameters and Potential Applications of Nanofibers in Biomedical and Biotechnology. Arabian J. Chem. 2018, 11, 1165-1188.

(34) Stachewicz, U.; Stone, C. A.; Willis, C. R.; Barber, A. H. Charge Assisted Tailoring of Chemical Functionality at Electrospun Nanofiber Surfaces. J. Mater. Chem. 2012, 22, 22935.

(35) Busolo, T.; Ura, D. P.; Kim, S. K.; Marzec, M. M.; Bernasik, A.; Stachewicz, U.; Kar-Narayan, S. Surface Potential Tailoring of PMMA Fibers by Electrospinning for Enhanced Triboelectric Performance. Nano Energy 2019, 57, 500-506.

(36) Baji, A.; Mai, Y.-W.; Li, Q.; Liu, Y. Electrospinning Induced Ferroelectricity in Poly(Vinylidene Fluoride) Fibers. Nanoscale 2011, 3, 3068-3071.

(37) Mohammadi, B.; Yousefi, A. A.; Bellah, S. M. Effect of Tensile Strain Rate and Elongation on Crystalline Structure and Piezoelectric Properties of PVDF Thin Films. Polym. Test. 2007, 26, 42-50.

(38) Stachewicz, U.; Szewczyk, P. K.; Kruk, A.; Barber, A. H.; Czyrska-Filemonowicz, A. Pore Shape and Size Dependence on Cell Growth into Electrospun Fiber Scaffolds for Tissue Engineering: 2D and 3D Analyses Using SEM and FIB-SEM Tomography. Mater. Sci. Eng., C 2019, 95, 397-408.

(39) Kalinin, S. V.; Rodriguez, B. J.; Jesse, S.; Shin, J.; Baddorf, A. P.; Gupta, P.; Jain, H.; Williams, D. B.; Gruverman, A. Vector Piezoresponse Force Microscopy. Microsc. Microanal. 2006, 12, 206-220.

(40) Soergel, E. Piezoresponse Force Microscopy (PFM). J. Phys. D: Appl. Phys. 2011, 44, 464003.

(41) Choi, Y. S.; Kim, S. K.; Williams, F.; Calahorra, Y.; Elliott, J. A.; Kar-Narayan, S. The Effect of Crystal Structure on the Electromechanical Properties of Piezoelectric Nylon-11 Nanowires. Chem. Commun. 2018, 54, 6863-6866.
(42) Richard-Lacroix, M.; Pellerin, C. Molecular Orientation in Electrospun Fibers: From Mats to Single Fibers. Macromolecules 2013, 46, 9473-9493.

(43) Kiselev, P.; Rosell-Llompart, J. Highly Aligned Electrospun Nanofibers by Elimination of the Whipping Motion. J. Appl. Polym. Sci. 2012, 125, 2433-2441.

(44) Pai, C.-L.; Boyce, M. C.; Rutledge, G. C. Morphology of Porous and Wrinkled Fibers of Polystyrene Electrospun from Dimethylformamide. Macromolecules 2009, 42, 2102-2114.

(45) Yazgan, G.; Dmitriev, R. I.; Tyagi, V.; Jenkins, J.; Rotaru, G.-M.; Rottmar, M.; Rossi, R. M.; Toncelli, C.; Papkovsky, D. B.; ManiuraWeber, K.; et al. Steering Surface Topographies of Electrospun Fibers: Understanding the Mechanisms. Sci. Rep. 2017, 7, 158.

(46) Reneker, D. H.; Yarin, A. L.; Zussman, E.; Xu, H. Electrospinning of Nanofibers from Polymer Solutions and Melts. Adv. Appl. Mech. 2007, 41, 43-346.

(47) Nezarati, R. M.; Eifert, M. B.; Cosgriff-Hernandez, E. Effects of Humidity and Solution Viscosity on Electrospun Fiber Morphology. Tissue Eng., Part C 2013, 19, 810-819.

(48) Cozza, E. S.; Monticelli, O.; Marsano, E.; Cebe, P. On the Electrospinning of PVDF: Influence of the Experimental Conditions on the Nanofiber Properties. Polym. Int. 2013, 62, 41-48.

(49) Dhakras, D.; Borkar, V.; Ogale, S.; Jog, J.; Nanoscale, C.; Dhakras, D.; Borkar, V.; Jog, J. Enhanced Piezoresponse of Electrospun PVDF Mats with a Touch of Nickel Chloride Hexahydrate Salt. Nanoscale 2012, 4, 752.

(50) Salimi, A.; Yousefi, A. A. Analysis Method. Polym. Test. 2003, 22, 699-704.

(51) Gregorio, R., Jr.; Cestari, M. Effect of Crystallization Temperature on the Crystalline Phase Content and Morphology of Poly(Vinylidene Fluoride). J. Polym. Sci., Part B: Polym. Phys. 1994, 32, 859-870.

(52) Gradys, A.; Sajkiewicz, P. Determination of the Melting Enthalpy of $\beta$ Phase of Poly(Vinylidene Fluoride). e-Polym. 2013, 13, 203-216.

(53) Yu, H.; Huang, T.; Lu, M.; Mao, M.; Zhang, Q.; Wang, H. Enhanced Power Output of an Electrospun PVDF/MWCNTs-Based Nanogenerator by Tuning Its Conductivity. Nanotechnology 2013, 24, 405401 .

(54) Fang, J.; Wang, X.; Lin, T. Electrical Power Generator from Randomly Oriented Electrospun Poly(Vinylidene Fluoride) Nanofibre Membranes. J. Mater. Chem. 2011, 21, 11088-11091. 